\title{
Selection of optimal pixel resolution for landslide susceptibility analysis within the Bukit Antarabangsa, Kuala Lumpur, by using image processing and multivariate statistical tools
}

\author{
lqbal Quraishi ${ }^{1 *}$, Abul Hasnat ${ }^{2}$ and J. Paul Choudhury ${ }^{1}$
}

\begin{abstract}
Landslides are considered as one of the natural hazards responsible for casualties, damage of assets, and infrastructures. In many situations, collection of field data from remote places is difficult due to inaccessibility of landslide area. This paper examines landslide susceptibility in the Bukit Antarabangsa, Kuala Lumpur, to ease geographical studies, using image processing and multivariate statistical tools by reviewing the digital images using remote-sensing technique without any physical survey. We considered different pixel resolutions and report the effectiveness of using factor analysis, principal component analysis, linear discriminant analysis, and their hybridization. Eight types of databases for heavy, medium, and no landslide were created. The modeling works were carried out at $2 \times 2,4 \times 4,8 \times 8,16 \times 16$, $32 \times 32,64 \times 64,128 \times 128$, and $256 \times 256$ pixel resolutions. Results indicate $2 \times 2$ was optimal in both heavy and medium while $8 \times 8$ found to be ideal for no landslide region. Performance at different pixel resolutions was compared using receiver operating characteristic (ROC) curves, and average success of $87.36 \%$ was found. This simple yet robust system holds great potential for saving lives.
\end{abstract}

Keywords: Landslide, Image processing, Factor analysis, Principal component analysis, Linear discriminant analysis, Residual analysis, Multivariate statistical tools

\section{Introduction}

Landslide is an extreme natural phenomenon that takes a heavy toll on human life and property leaving farreaching consequence not only on economy but also nature and ecosystem of the affected region. Flash flood, long and terrible monsoon, earthquake rock sliding or toppling, soil cave-in, and sudden profusion of snow melting are some of the elementals that precede landslide in a particular area. Again, soil condition alters after earthquake or any other natural transformation in a region and its neighborhood making it more vulnerable to landslide. Landslide susceptibility analysis can be helpful in such case as certain preventive measures can

\footnotetext{
* Correspondence: iqbalqu@gmail.com

'Department of Information Technology, Kalyani Government Engineering College, Kalyani, Nadia, West Bengal, India

Full list of author information is available at the end of the article
}

be taken in time to minimize future threat to human life in the best possible way. Tarantino et al. [1] had applied change detection techniques for monitoring landslides in southern Italy. Saha et al. [2] had utilized geographic information system (GIS)-based statistical approach for landslide susceptibility in the Himalayas. Liang et al. [3] had used multi-satellite images and GIS data for statistical analysis of landslide in Taiwan Island. Rau et al. [4] had applied time series satellite images for monitoring and assessment of landslide. Voigt et al. [5] had shown the efficient use of image analysis based on satellite images for landslide mapping. Joyce et al. [6] had also used image processing techniques with manual interpretation for predicting landslide proneness. Rainfall recording data had been taken by Martelloni et al. [7] for the prediction of landslide in a local territory of Emilia Romagna, Italy. Kanungo and Sharma [8] had 


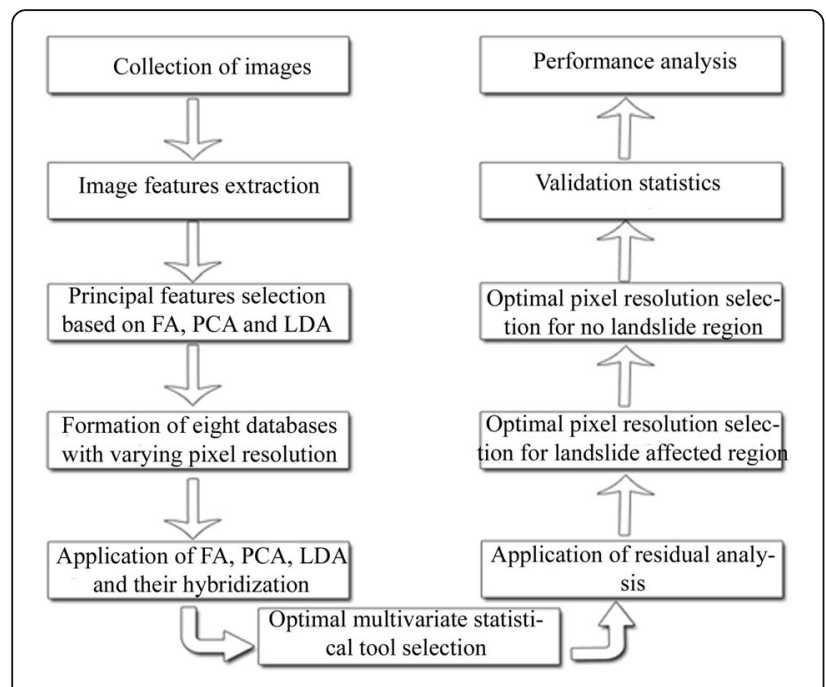

Fig. 1 Block diagram of the proposed system

obtained rainfall threshold values for landslides in and around Chamoli, Joshimath region of the Garhwal Himalayas, India. Cultivated, fallow, and wood land data had been investigated by Biro et al. [9] for landslide assessment. Topological, geological, and environmental parameters are used as parameters for landslide assessment by Akgun [10]. Aerial photographs and field surveys of Cameron Highlands, Malaysia, had been utilized by Pradhan et al. [11] to analyze landslide hazards, and accuracy of $83.45 \%$ was established. Pradhan et al. [12] have also used spatial-based statistical models for landslide hazard analysis. Pradhan et al. [13] developed a neuro-fuzzy model using remote-sensing data and GIS in a part of the Cameron Highland areas in Malaysia. GIS and remote-sensing data were used by Lee et al. [14, 15] for the assessment of landslide. Historical data of rainfall and earthquake was taken by Muthu et al. [16] for landslide analysis. Assessment of landslide using lithology, rock weathering, geomorphology, soil type, and depth had been conducted by Champatiray et al. [17]. It is observed that most of the authors have used field data like

Table 1 Pixel resolution and their dimensions

\begin{tabular}{lllll}
\hline Databases & $\begin{array}{l}\text { Pixel } \\
\text { resolution }\end{array}$ & $\begin{array}{l}\text { Width } \\
\text { in pixels }\end{array}$ & $\begin{array}{l}\text { Height } \\
\text { in pixels }\end{array}$ & $\begin{array}{l}\text { Total number } \\
\text { of pixels }\end{array}$ \\
\hline Images for database I & $2 \times 2$ & 2 & 2 & 4 \\
Images for database II & $4 \times 4$ & 4 & 4 & 16 \\
Images for database III & $8 \times 8$ & 8 & 8 & 64 \\
Images for database IV & $16 \times 16$ & 16 & 16 & 256 \\
Images for database V & $32 \times 32$ & 32 & 32 & 1024 \\
Images for database VI & $64 \times 64$ & 64 & 64 & 4096 \\
Images for database VII & $128 \times 128$ & 128 & 128 & 16,384 \\
Images for database VIII & $256 \times 256$ & 256 & 256 & 65,536 \\
\hline
\end{tabular}

Table 2 Image features and their description

\begin{tabular}{|c|c|c|}
\hline Feature & $\begin{array}{l}\text { No. of } \\
\text { Features }\end{array}$ & Details \\
\hline Fractal dimension & 3 & $\begin{array}{l}\text { Mean of fractal dimension, standard } \\
\text { deviation of fractal dimension, } \\
\text { lacunarity }\end{array}$ \\
\hline Ripplet coefficient & 2 & $\begin{array}{l}\text { Mean of ripplet coefficient, standard } \\
\text { deviation of ripplet coefficient }\end{array}$ \\
\hline $\begin{array}{l}\text { Autocorrelation } \\
\text { coefficient }\end{array}$ & 2 & $\begin{array}{l}\text { Mean of autocorrelation coefficient, } \\
\text { standard deviation of autocorrelation } \\
\text { coefficient }\end{array}$ \\
\hline $\begin{array}{l}\text { Gray level } \\
\text { co-occurrence } \\
\text { matrix }\end{array}$ & 16 & $\begin{array}{l}\text { Four set of data values; each set consist } \\
\text { of the contrast, homogeneity, energy, } \\
\text { and correlation }\end{array}$ \\
\hline $\begin{array}{l}\text { Gray level run } \\
\text { length matrix }\end{array}$ & 44 & $\begin{array}{l}\text { Short run emphasis, long run emphasis, } \\
\text { gray level non-uniformity, run length } \\
\text { non-uniformity, run percentage, low } \\
\text { gray level run emphasis, high gray } \\
\text { level run emphasis, short run low } \\
\text { gray level emphasis, short run high } \\
\text { gray level emphasis, long run low } \\
\text { gray level emphasis, long run high } \\
\text { gray level emphasis. Each of the } \\
\text { above was calculated for } 0,45,90 \text {, } \\
\text { and } 135\end{array}$ \\
\hline Gabor coefficient & 60 & $\begin{array}{l}\text { Mean and standard deviation of } \\
\text { Gabor coefficients for } 0,15,30,45 \text {, } \\
60,75\end{array}$ \\
\hline Moments & 4 & $\begin{array}{l}\text { First moment, second moment, } \\
\text { third moment, and fourth moment }\end{array}$ \\
\hline
\end{tabular}

geological and topological parameters, rainfall, and their likes in addition to the satellite image information of that place for the assessment of landslide susceptibility.

The objective of our research work is to analyze and asses the landslide susceptibility based on the examination of the satellite images of a particular region. It must be borne in mind the image feature values of a landslide-affected area are different from those of the

Table 3 Multivariate statistical tools and their hybridized variants

\begin{tabular}{|c|c|c|}
\hline No. & Method/tools & Description \\
\hline 1. & FA & Factor analysis \\
\hline 2. & $F A+P C A$ & $\begin{array}{l}\text { Factor analysis followed by principal } \\
\text { component analysis }\end{array}$ \\
\hline 3. & $F A+L D A$ & $\begin{array}{l}\text { Factor analysis followed by linear } \\
\text { discriminant analysis }\end{array}$ \\
\hline 4. & LDA & Linear discriminant analysis \\
\hline 5. & $\mathrm{LDA}+\mathrm{FA}$ & $\begin{array}{l}\text { Linear discriminant analysis followed } \\
\text { by factor analysis }\end{array}$ \\
\hline 6. & $L D A+P C A$ & $\begin{array}{l}\text { Linear discriminant analysis followed } \\
\text { by principal component analysis }\end{array}$ \\
\hline 7. & PCA & Principal component analysis \\
\hline 8. & $P C A+F A$ & $\begin{array}{l}\text { Principal component analysis } \\
\text { followed by factor analysis }\end{array}$ \\
\hline 9. & $P C A+L D A$ & $\begin{array}{l}\text { Principal component analysis followed } \\
\text { by linear discriminant analysis }\end{array}$ \\
\hline
\end{tabular}


Table 4 Confusion matrix

\begin{tabular}{llll}
\hline & & \multicolumn{2}{l}{ Landslide occurrence } \\
\cline { 3 - 4 } & & Occurred (yes) & Occurred (no) \\
\hline Landslide & Predicted (yes) & True positive (TP) & False positive (FP) \\
prediction & Predicted (no) & False negative (FN) & True negative (FN) \\
\hline
\end{tabular}

normal one. Examination of these changes in such parameters using image processing and multivariate statistical tools may hold the key. The main task in our research work constitutes the collection of image features; selection of principal features out of 131 image features; formation of eight databases with varying pixel resolution for heavy, medium, and no landslide-affected regions; selection of optimal pixel resolution out of $2 \times$ $2,4 \times 4,8 \times 8,16 \times 16,32 \times 32,64 \times 64,128 \times 128$, and $256 \times 256$ for heavy, medium, and no landslide-affected regions, respectively, based on residual analysis, multivariate statistical tools, and their hybridized ones. Finally, analysis of performance, testing, and validation of the experimental work is carried out.

\section{Methods}

\subsection{Data collection and database creation}

A sequence of main processing phases proposed is furnished in Fig. 1. Eight types of databases are created for heavy, medium, and no landslide-affected regions, depending on the condition of soil. Each database consists of 450 images of pixel resolution $2 \times 2,4 \times 4,8 \times 8,16 \times$ $16,32 \times 32,64 \times 64,128 \times 128$, and $256 \times 256$ respectively. The pixel resolution $2 \times 2$ indicates the length and breadth of the image is 2 pixels. The detailed description of image databases with varying pixel resolution is presented in Table 1.

Different pixel resolutions are considered for feature extraction. Li et al. [18] had estimated fractal dimension (FD) using box-counting approach as an important feature for texture description in an image. Whereas Hawlick et al. [19] reported a survey for various statistical and structural features for image texture classification like autocorrelation function, optical transform, digital transform, textural edgeness, run length, gray tone co-occurrence, structural element, and autoregressive models. Thangavel and Manavalan [20] applied gray level co-occurrence matrix (GLCM) features for image classification of prostate cancer. Variance, kurtosis, skewness, and geometric mean are used as features for fault diagnosis of ball bearing by Vakharia et al. [21]. Fernandez-Lozano et al. [22] used statistical and run length features for texture classification. Mercimek et al. [23] proposed moments as invariant global features of images in pattern recognition. Filho et al. [24] measured lacunarity using Gliding-Box and Differential Box-Counting

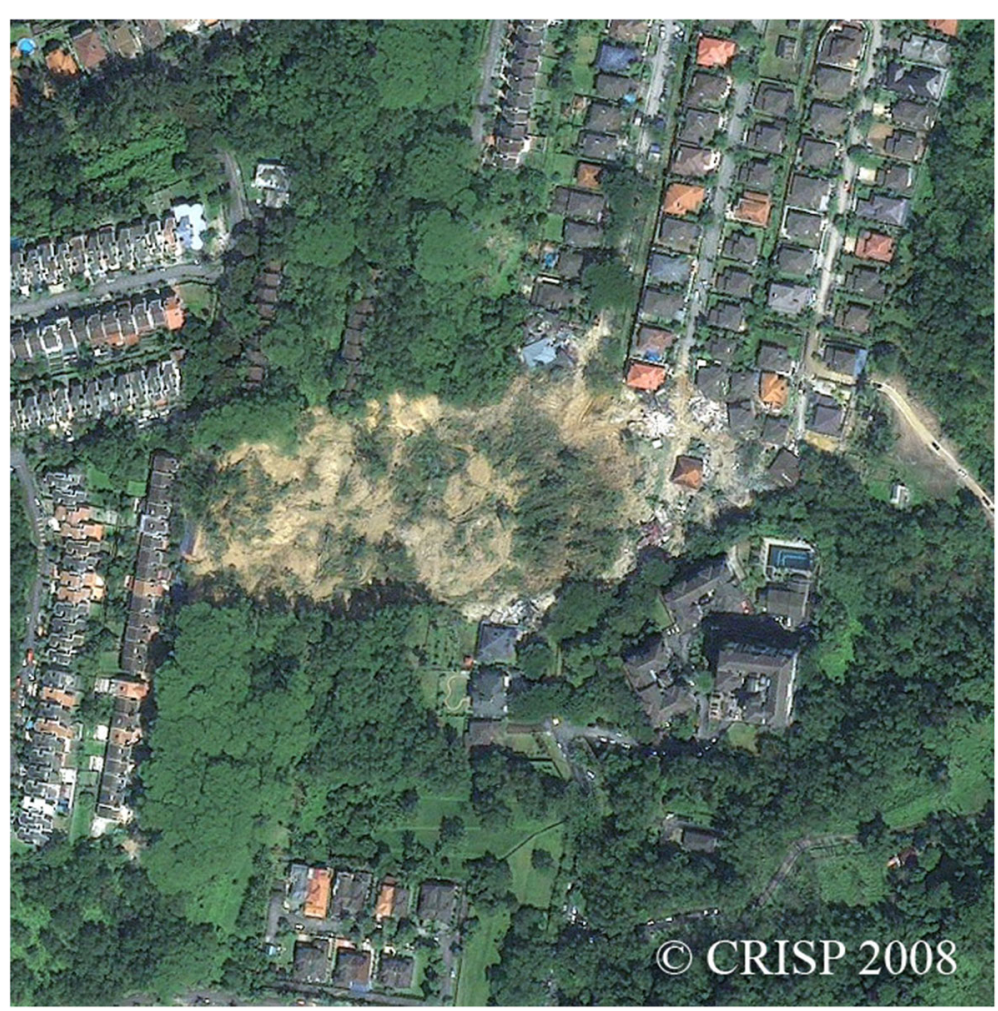

Fig. 2 Image of north-eastern side of Bukit Antarabangsa, Kuala Lumpur, collected on 9 December 2008, after landslide 

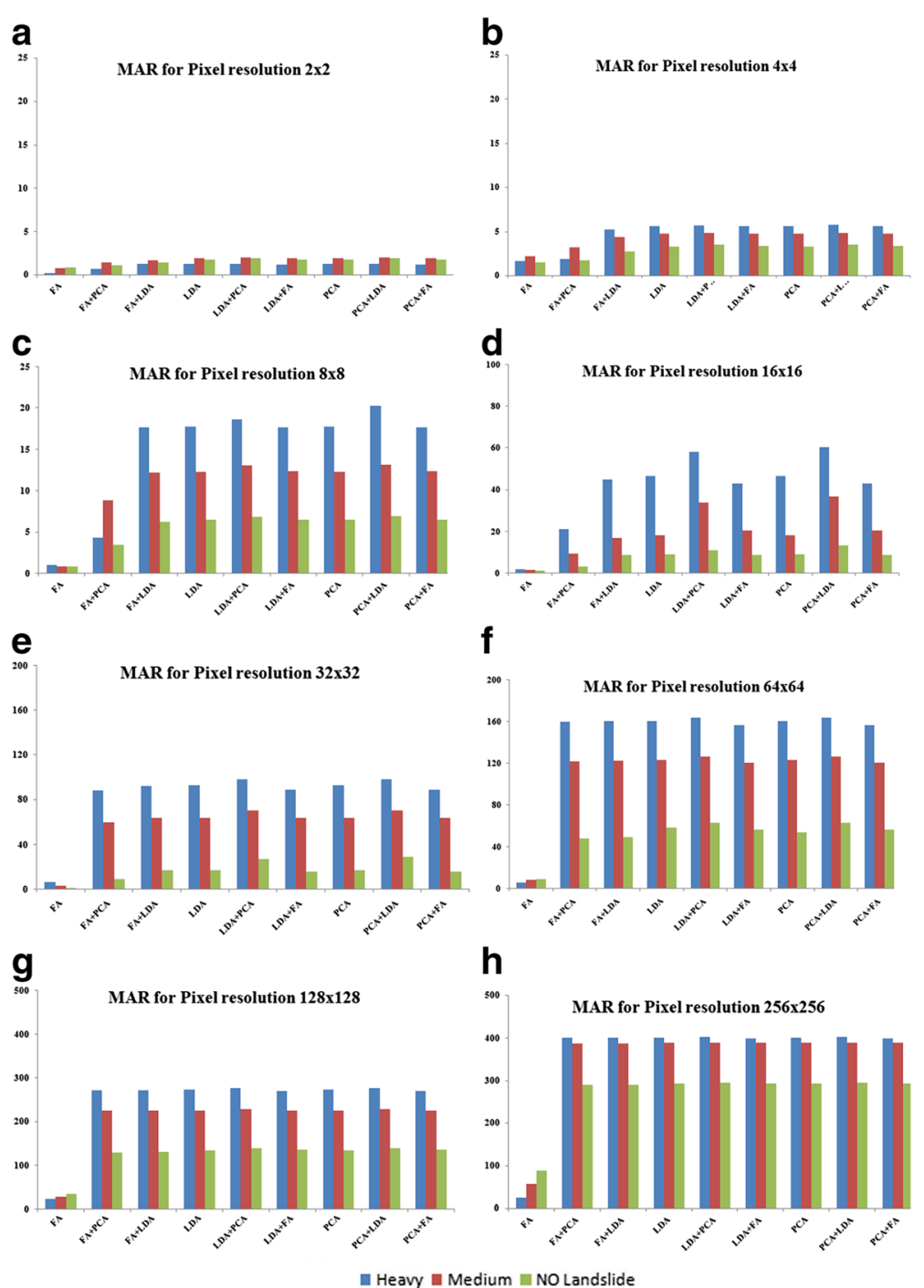

Fig. 3 Variation of values of MAR ( $Y$-axis) against different multivariate statistical tools ( $X$-axis). a $2 \times 2, \mathbf{b} 4 \times 4, \mathbf{c} 8 \times 8$, d $16 \times 16$, e $32 \times 32$, f $64 \times 64, \mathbf{g} 128 \times 128$, and $\mathbf{h} 256 \times 256$ pixel resolutions. Blue, red, and green colors represent heavy, medium, and no landslide respectively

algorithms to classify textures of urban regions. Fernando et al. [25] suggested a scheme for mining mid-level features based on Frequent Local Histograms for image classification. Lowe [26] used fast nearest-neighbor algorithm followed by a Hough transform for feature extraction. In this study, various statistical characteristics of images, viz., mean of fractal dimension, standard deviation of fractal dimension, lacunarity, mean of ripplet coefficient, standard deviation of ripplet coefficient, mean of autocorrelation coefficient, and standard deviation of autocorrelation coefficient, are used for feature extraction of each image in eight databases. A total of 131 feature values are calculated. The details of features are presented in Table 2.

Out of 450 images taken into consideration for each database, 360 images are used to create the training dataset and the remaining 90 images are used for test purpose.

\subsection{Application of multivariate statistical tools and hybridization}

Multivariate statistical tools, viz., factor analysis, principal component analysis, and linear discriminant analysis are briefly discussed below.

\subsubsection{Factor analysis (FA)}

FA [27, 28] is a statistical method used to describe variability among observed, correlated variables in terms of a potentially lower number of unobserved variables called factors. Initially, the correlation matrix and the contribution of eigenvectors and eigenvalues are calculated. The eigenvector with the 

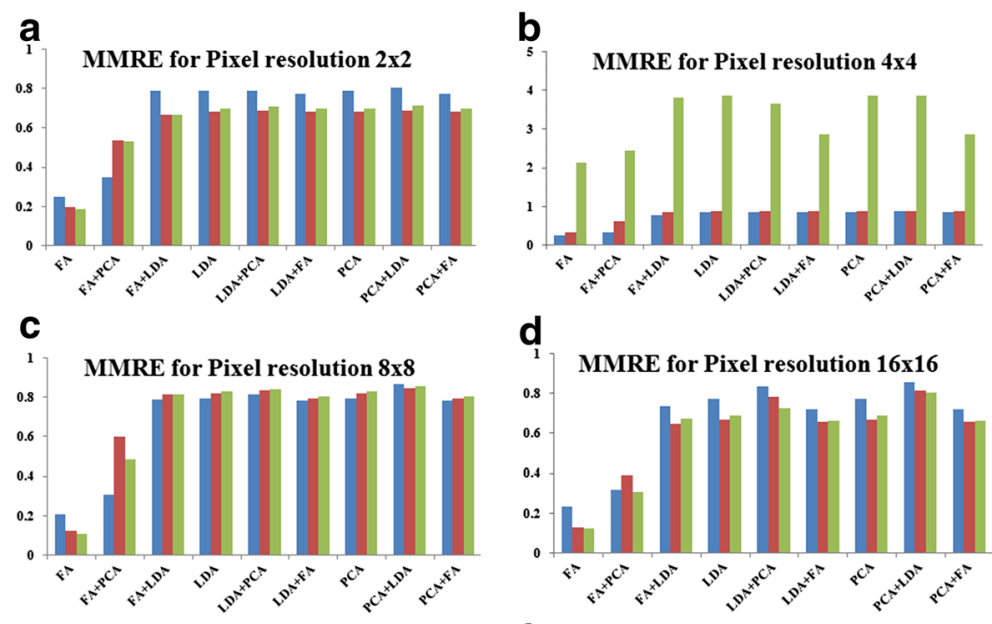

\section{d}
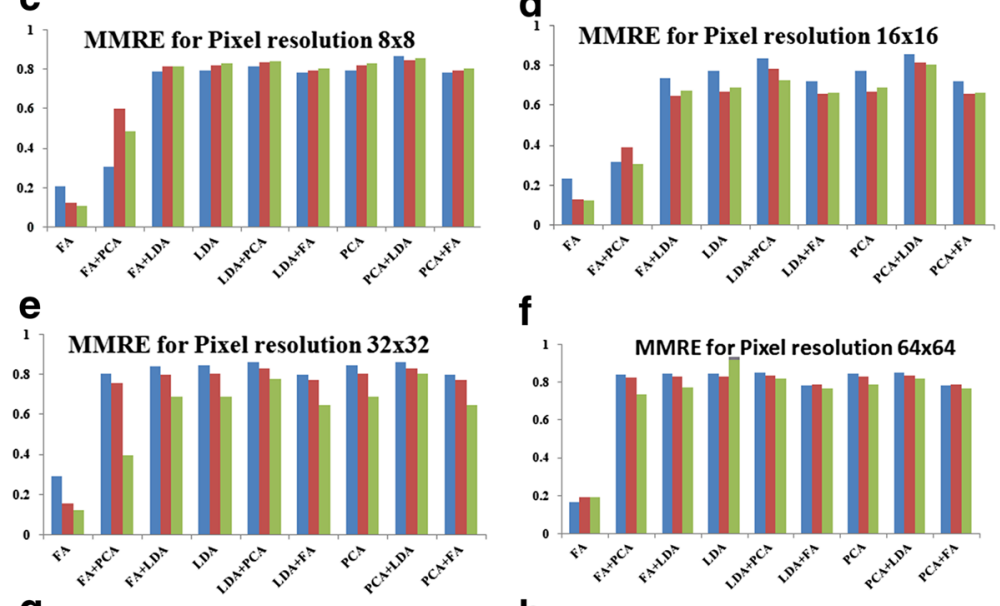

f

g
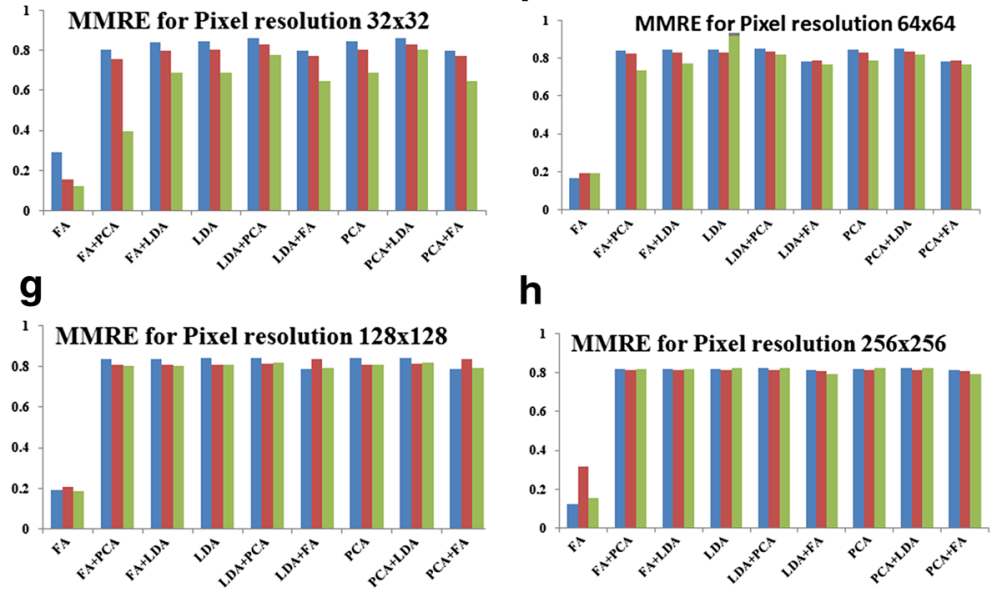

h

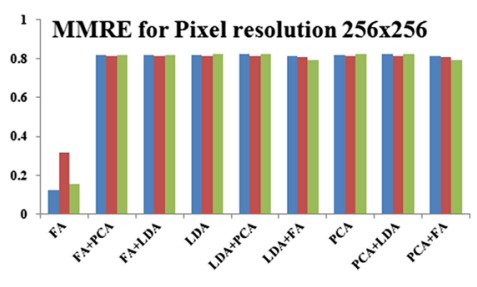

Fig. 4 Variation of values of MMRE ( $Y$-axis) against different multivariate statistical tools ( $X$-axis). a $2 \times 2$, b $4 \times 4$, c $8 \times 8$, d $16 \times 16$, e $32 \times 32$, $\mathbf{f} 64 \times 64, \mathbf{g} 128 \times 128$, and $\mathbf{h} 256 \times 256$ pixel resolutions. Blue, red, and green colors represent heavy, medium, and no landslide respectively

highest eigenvalues is the major component of the dataset [27, 28]. Then the lower important dimensions contributing less than $2.5 \%$ are discarded. The square root of the corresponding eigenvalues are taken and multiplied with the square of the eigenvector to get the column-wise contributor for each of the major contributing component. At the last step, the ranges of cumulative values for each database are calculated.

\subsubsection{Principal component analysis ( $P C A)$}

PCA [29] is used to recognize patterns in data of high dimension. At first, the mean of the dataset is calculated and subtracted from each of the data dimensions. Then, the covariance matrix, eigenvectors, and eigenvalues are calculated. The lower important dimensions contributing less than $2.5 \%$ are discarded. The corresponding eigenvalues are multiplied with the square of the eigenvector to get the column-wise contributor for each of the major contributing components. The ranges of cumulative values for each database are calculated.

\subsubsection{Linear discriminant analysis (LDA)}

LDA [30] maximizes the ratio of between-class variance to the within-class variance in any dataset. The major dissimilarity between LDA and PCA is that, generally, PCA is used for feature classification, whereas LDA is used for data classification. Also, in the case of PCA, when dataset is transformed to another space, position of the original dataset changes, whereas in the case of LDA, the position remains the same. For LDA, initially, the corresponding mean for each component are calculated and subtracted from the original dataset to create a new dataset. Then, the new dataset and its transpose are multiplied to 


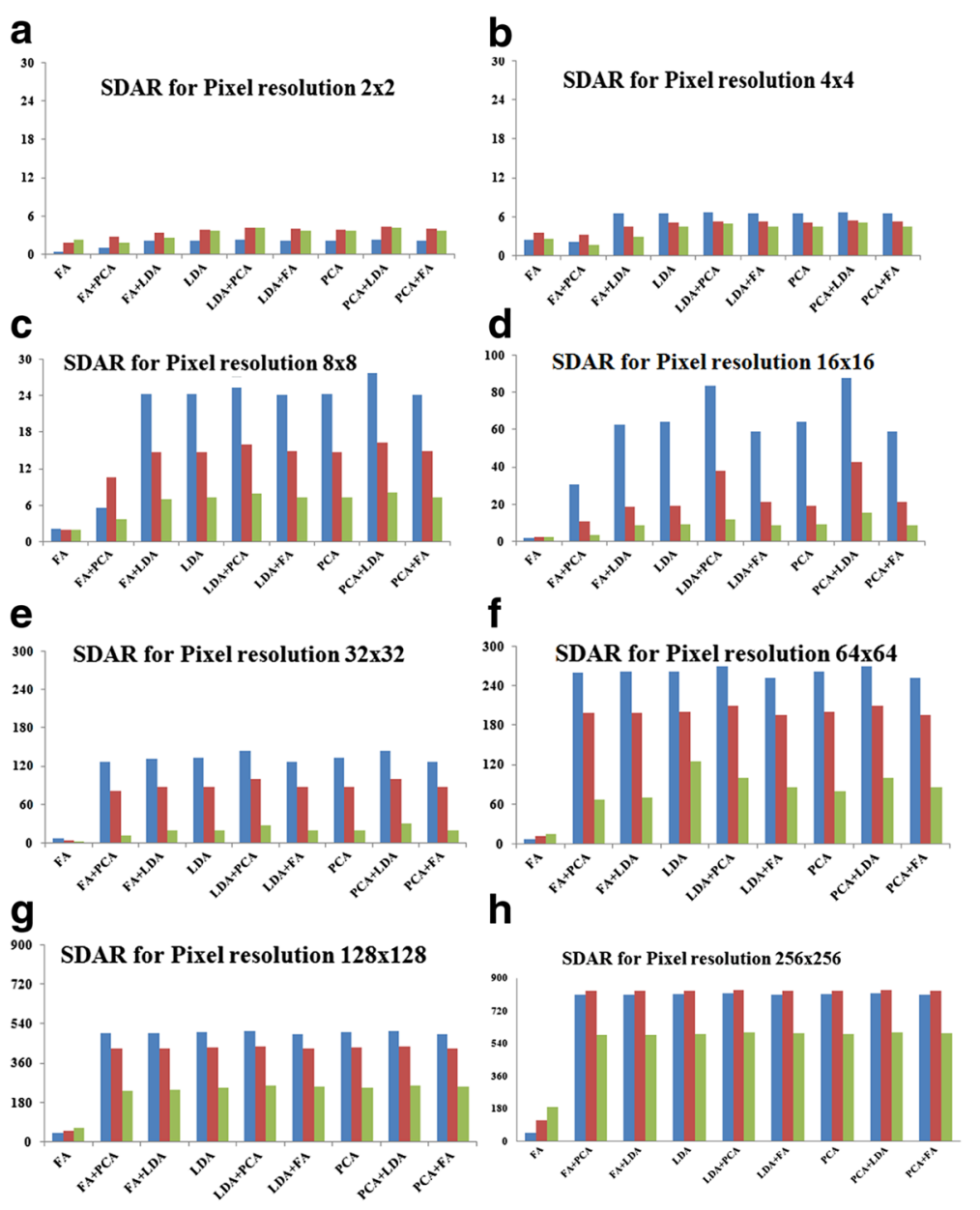

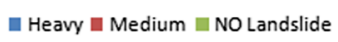

Fig. 5 Variation of values of SDAR (Y-axis) against different multivariate statistical tools (X-axis). (a) $2 \times 2$, (b) $4 \times 4$, (c) $8 \times 8$, (d) $16 \times 16$, (e) $32 \times 32$, (f) $64 \times 64$, (g) $128 \times 128$, (h) $256 \times 256$ pixel resolutions. Blue, red and green colors represent heavy, medium and no landslide respectively

create the modified matrix. Next, contributions of eigenvectors among all the eigenvalues are calculated. The lower important dimensions contributing less than $2.5 \%$ are discarded. The corresponding eigenvalues are taken and multiplied with the square of

Table 5 Values of MAR, MMRE, and SDAR for heavy landslide prone regions using FA

\begin{tabular}{llll}
\hline Pixel resolution & MAR & MMRE & SDAR \\
\hline $2 \times 2$ & 0.206782785 & 0.252547158 & 0.442549705 \\
$4 \times 4$ & 1.662994379 & 0.263329912 & 2.50564727 \\
$8 \times 8$ & 1.047158087 & 0.211043584 & 2.19255461 \\
$16 \times 16$ & 1.847208125 & 0.237919993 & 2.054714319 \\
$32 \times 32$ & 6.70861389 & 0.293023533 & 7.852577432 \\
$64 \times 64$ & 5.748555656 & 0.168775894 & 7.863123912 \\
$128 \times 128$ & 23.5687214 & 0.190467907 & 41.43065804 \\
$256 \times 256$ & 24.55296951 & 0.122525748 & 46.07557327 \\
\hline
\end{tabular}

the eigenvectors to get the column-wise contributor for each of the major contributing component. The range of cumulative values for each database is calculated. The hybridized variant of FA, PCA, and LDA are applied also as shown in Table 3.

Table 6 Values of MAR, MMRE, and SDAR for medium landslide prone regions using FA

\begin{tabular}{llll}
\hline Pixel resolution & MAR & MMRE & SDAR \\
\hline $2 \times 2$ & 0.761354656 & 0.197167578 & 1.933125496 \\
$4 \times 4$ & 2.140939402 & 0.336082884 & 3.581477082 \\
$8 \times 8$ & 0.899307899 & 0.12736707 & 2.001307805 \\
$16 \times 16$ & 1.49834217 & 0.131642179 & 2.627122352 \\
$32 \times 32$ & 3.260209915 & 0.155194255 & 3.835789063 \\
$64 \times 64$ & 8.523614596 & 0.191849412 & 12.44882429 \\
$128 \times 128$ & 27.65028247 & 0.208976205 & 52.43451591 \\
$256 \times 256$ & 57.87334771 & 0.320894902 & 118.2940963 \\
\hline
\end{tabular}


Table 7 Values of MAR, MMRE, and SDAR for no landslide prone regions using FA

\begin{tabular}{llll}
\hline Pixel resolution & MAR & MMRE & SDAR \\
\hline $2 \times 2$ & 0.861486797 & 0.185372755 & 2.256215954 \\
$4 \times 4$ & 1.48792689 & 2.143451896 & 2.723227198 \\
$8 \times 8$ & 0.895715057 & 0.111072934 & 2.036777743 \\
$16 \times 16$ & 1.258698993 & 0.128076209 & 2.60819919 \\
$32 \times 32$ & 1.248390761 & 0.126545613 & 2.529666286 \\
$64 \times 64$ & 8.832916851 & 0.195855956 & 14.89904491 \\
$128 \times 128$ & 33.91913036 & 0.188469583 & 64.86457402 \\
$256 \times 256$ & 87.53532343 & 0.156648294 & 189.7140945 \\
\hline
\end{tabular}

\subsection{Residual analysis}

Residual analysis is done to assess the performance of the multivariate statistical tools and their hybridized variants while applied on all the databases. The parameters used in this study for performance evaluation are absolute residual (AR), mean of absolute residual (MAR), standard deviation of absolute residual (SDAR), mean residual error (MRE), and mean of mean residual error (MMRE) [31, 32]. The smaller value of these parameters of residual analysis indicates better model. The different parameters are defined using Eqs. 1-5.

Absolute Residual, $\operatorname{AR}(i, j)=|\operatorname{actual}(i, j)-\operatorname{appr}(i, j)|$

Mean of Absolute Residual (MAR) $=\frac{\sum_{i=1}^{m} \sum_{j=1}^{n} \operatorname{AR}(i, j)}{m * n}$

Standard Deviation of Absolute Residual (SDAR)

$$
=\sqrt{\frac{\sum_{i=1}^{m} \sum_{j=1}^{n}(\mathrm{AR}(i, j)-\mathrm{MAR})^{2}}{m * n}}
$$

Mean of Residual Error, $\operatorname{MRE}(i, j)=\frac{\mid \operatorname{actual}(i, j)-\operatorname{appr}(i, j)}{\operatorname{actual}(i, j)}$

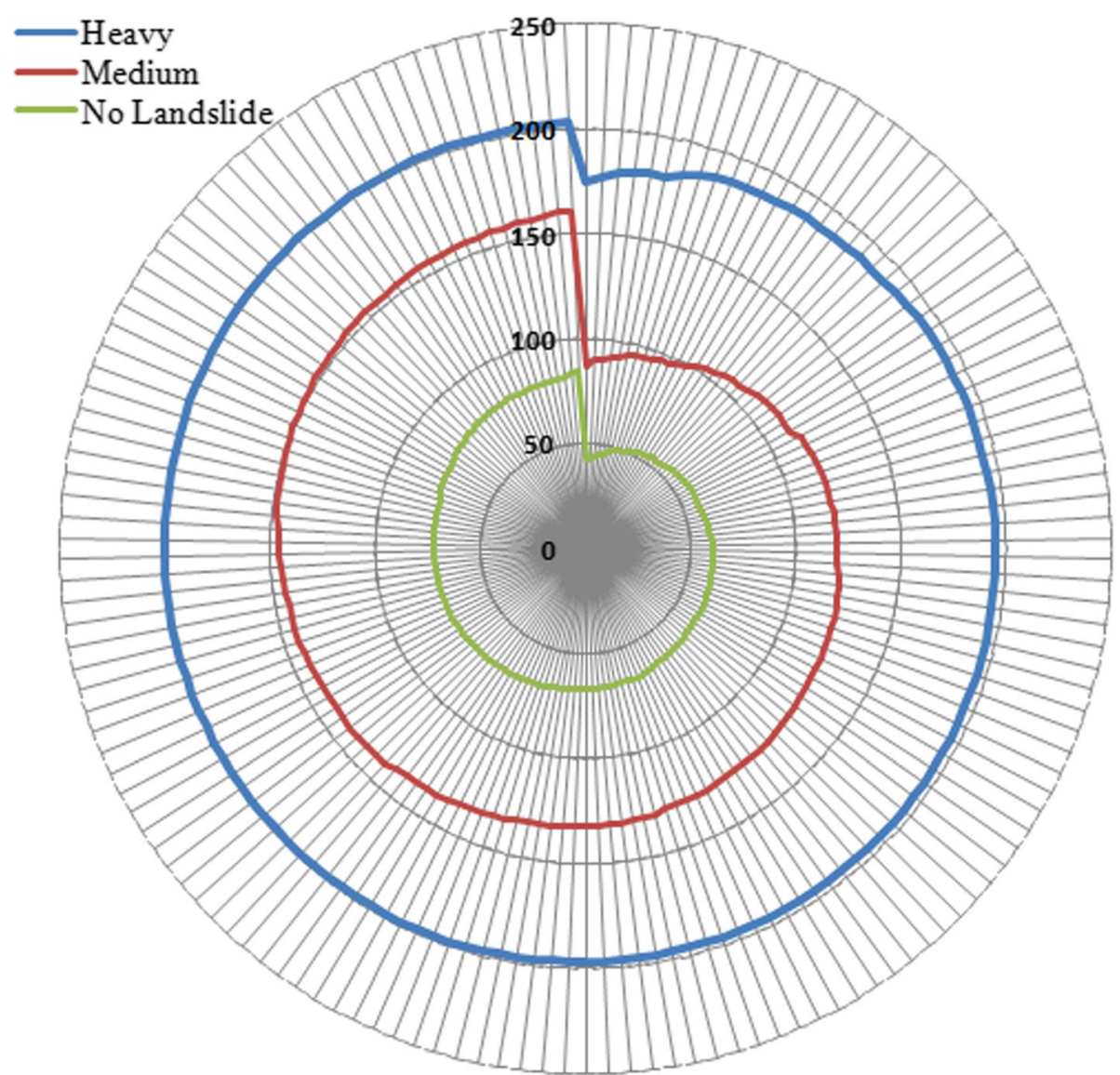

Fig. 6 Variation of cumulative values (Y-axis) against $2 \times 2$ pixel resolution image samples (X-axis) after application of FA. Blue, red, and green colors represent heavy, medium, and no landslide respectively 
Table 8 Range of cumulative values for different types of $2 \times 2$ pixel resolution images using FA

\begin{tabular}{lcc}
\hline Image type & Minimum cumulative value & Maximum cumulative value \\
\hline Heavy & 174.3266 & 203.1112 \\
Medium & 87.3197 & 161.3021 \\
No landslide & 43.0205 & 85.2026 \\
\hline
\end{tabular}

$$
\text { Mean of } \operatorname{MRE}(\mathrm{MMRE})=\frac{\sum_{i=1}^{m} \sum_{j=1}^{n} \operatorname{MRE}(i, j)}{m * n}
$$

Where actual $(i, j)$ and $\operatorname{appr}(i, j)$ are $(i, j)$ th element in the true value matrix and observed value matrix having $m$ rows and $n$ columns respectively.

\subsection{Validation statistics}

True positive (TP) values are the incidents where models have correctly predicted landslide. False positive (FP) or false alarms are the situation where the models predicted landslide but, actually, landslide has not occurred. False negative (FN) or missed alarms are the instances where the models predicted no landslide but, actually, landslide has occurred. True negative (TN) values are the incidents where the model has correctly predicted no landslide. Correct prediction (true positive and true negative) and errors (false negative and false positive) are calculated. These terms are defined by a confusion matrix as given in Table 4.

The validation statistics, viz., efficiency, misclassification rate, positive predictive power, negative predictive power, sensitivity, specificity, false positive rate, and false negative rate, are defined using Eqs. 6-13.

$$
\begin{aligned}
& \text { Efficiency }=\frac{\mathrm{TP}+\mathrm{TN}}{\mathrm{TP}+\mathrm{TN}+\mathrm{FP}+\mathrm{FN}} \\
& \text { Misclassification Rate }=\frac{\mathrm{FP}+\mathrm{FN}}{(\mathrm{TP}+\mathrm{TN}+\mathrm{FP}+\mathrm{FN})}
\end{aligned}
$$

Table 9 Matching and alarm statistics

\begin{tabular}{lllllllllllllll}
\hline Exp no. & Pixel resolution & TP & \multicolumn{1}{ll}{ TN } & \multicolumn{1}{c}{ FP } & \multicolumn{1}{c}{ FN } \\
\hline & & $H$ & $M$ & $N$ & $H$ & $M$ & $N$ & $H$ & $M$ & $N$ & $H$ & $M$ & $N$ \\
1 & $2 \times 2$ & 15 & 8 & 7 & 20 & 11 & 22 & 1 & 1 & 2 & 1 & 1 & 1 \\
2 & $4 \times 4$ & 17 & 5 & 13 & 22 & 10 & 13 & 1 & 2 & 1 & 2 & 2 & 2 \\
3 & $8 \times 8$ & 10 & 7 & 8 & 19 & 9 & 26 & 3 & 1 & 2 & 1 & 2 & 2 \\
4 & $16 \times 16$ & 16 & 8 & 12 & 18 & 9 & 15 & 4 & 3 & 2 & 1 & 1 & 1 \\
5 & $32 \times 32$ & 12 & 8 & 12 & 17 & 15 & 13 & 1 & 1 & 2 & 3 & 2 & 4 \\
6 & $64 \times 64$ & 9 & 7 & 10 & 13 & 13 & 23 & 3 & 2 & 2 & 3 & 1 & 4 \\
7 & $128 \times 128$ & 11 & 7 & 12 & 12 & 10 & 26 & 2 & 4 & 2 & 1 & 1 & 2 \\
8 & $256 \times 256$ & 13 & 8 & 17 & 15 & 12 & 14 & 3 & 1 & 2 & 1 & 2 & 2 \\
\hline
\end{tabular}

Note: $H, M$, and $N$ represents heavy, medium, and no landslide cases respectively

$$
\begin{aligned}
& \text { Positive Predictive Power }=\frac{\mathrm{TP}}{\mathrm{TP}+\mathrm{FP}} \\
& \text { Negative Predictive Power }=\frac{\mathrm{TN}}{\mathrm{FN}+\mathrm{TN}} \\
& \text { Sensitivity }=\frac{\mathrm{TP}}{\mathrm{TP}+\mathrm{FN}} \\
& \text { Specificity }=\frac{\mathrm{TN}}{\mathrm{FP}+\mathrm{TN}}
\end{aligned}
$$$$
\text { False Positive Rate }=\frac{\mathrm{FP}}{\mathrm{FP}+\mathrm{TN}}
$$$$
\text { False Negative Rate }=\frac{\text { FN }}{\mathrm{TP}+\mathrm{FN}}
$$

Sensitivity, specificity, and receiver operating characteristic (ROC) are used to measure the superiority and trustworthiness of an assessment. Sensitivity assesses the superiority of the assessment by identifying positive landslide. Whereas specificity calculates approximately the possibility of a region without landslide would be acceptably discarded. ROC curve is a graphic arrangement to display the relationship between sensitivity and specificity, and it assists for best model selection by deciding the optimal threshold for the landslide assessment. Positive predictive power is the percentage of regions with a positive assessment which actually have the landslide. Negative predictive power is the percentage of regions with a negative assessment which do not have the landslide. Positive and negative predictive powers are directly related to the prevalence of the landslide in the regions.

\section{Results and discussions}

\subsection{Data collection}

A landslide took place at Taman Bukit Mewah, Bukit Antarabangsa, Hulu Kelang, Selangor, Kuala Lumpur, Malaysia, at 3:30 a.m. measuring $109 \mathrm{~m}$ in width, $120 \mathrm{~m}$ in length, and $15 \mathrm{~m}$ in depth. About $101,500 \mathrm{~m}^{3}$ of ground had moved. It had totally obstructed the only communicating road, Jalan Bukit Antarabangsa, with neighborhood. The satellite images after landslide of Bukit Antarabangsa were collected from Ikonos Satellite Image @Centre for Remote Imaging, Sensing and Processing, National University of Singapore via http://www.crisp.nus.edu.sg [33]. Figure 2 shows the place at Bukit Antarabangsa on the north-eastern side of Kuala Lumpur collected on 9 December 2008, after landslide. It is situated at latitude 3 $9^{\prime} 58.94^{\prime \prime} N$ and longitude $101^{\circ} 45^{\prime} 33.392^{\prime \prime} E$. The image shows the source, track, and expanded toe of the landslide. The lower part of the landslide buried a number of houses, damaged other objects, and carried parts of buildings with down slope. Low et al. [34] carried out detailed analysis of Bukit Antarabangsa landslide and concluded prolonged rainfall as the main causal factor. 
Table 10 Validation results

\begin{tabular}{lllllllllll}
\hline SN & Attributes & $2 \times$ & $4 \times$ & $8 \times$ & $16 \times$ & $32 \times$ & $64 \times$ & $128 \times$ & $256 \times$ & Average \\
S No. & & 2 & 4 & 8 & 16 & 32 & 64 & 128 & 256 & \\
\hline 1 & TP & 30 & 35 & 25 & 36 & 32 & 26 & 30 & 38 & 30.75 \\
2 & TN & 53 & 45 & 54 & 42 & 45 & 49 & 48 & 41 & 47.13 \\
3 & FP & 4 & 4 & 6 & 9 & 4 & 7 & 8 & 6 & 6.25 \\
4 & FN & 3 & 6 & 5 & 3 & 9 & 8 & 4 & 5 & 5.87 \\
\hline
\end{tabular}

\subsubsection{Residual analysis}

The values of MAR, MMRE, and SDAR for the three types of images of heavy landslide prone region, medium landslide prone region, or no landslide prone region are calculated and shown in Figs. $3 \mathrm{a}-\mathrm{h}, 4 \mathrm{a}-\mathrm{h}$, and $5(\mathrm{a}-\mathrm{h}) \mathrm{re}-$ spectively. It is observed that in all the three cases, the values obtained using factor analysis is better than that of the other multivariate statistical tools or their hybridized variants. So, all the experimental results shown here are calculated using factor analysis only. Best pixel resolution among $2 \times 2-256 \times 256$, the values of MAR is selected, and MMRE and SDAR are calculated using factor analysis only (Tables 5, 6 and 7) for three types of images. In the case of heavy landslide prone region, it is found (Table 5) that out of three parameters of residual analysis, pixel resolution of $2 \times 2$ gives better result in terms of MAR and SDAR, while pixel resolution of $256 \times 256$ gives better result in terms of MMRE. Therefore, pixel resolution of $2 \times 2$ becomes ideal for heavy landslide prone region. In the case of medium landslide prone region (Table 6), pixel resolution of $2 \times 2$ gives better result in terms of MAR and SDAR, while pixel resolution of $8 \times 8$ gives better result in terms of MMRE. Therefore, pixel resolution of $2 \times$ 2 is ideal for medium landslide prone region also.

In the case of no landslide prone region (Table 7), the pixel resolution of $8 \times 8$ gives better result in terms of MMRE and SDAR, while pixel resolution of $2 \times 2$ gives better result in terms of MAR. Therefore, pixel resolution of $8 \times 8$ becomes ideal for no landslide prone region. The pixel resolution of $2 \times 2$ is suitable in heavy and medium landslide prone region, while pixel resolution of $8 \times 8$ is suitable for no landslide prone region. So, pixel resolution of $2 \times 2$ can be considered ideal for landslide images (heavy and medium). The variation of cumulative values of $2 \times 2$ pixel resolution images of heavy, medium, and no landslide affection after application of FA is shown in Fig. 6. It is found that the range of cumulative values for heavy, medium, and no landslide affection are well separated.

Table 11 Prediction accuracy

\begin{tabular}{llllllllll}
\hline Attributes & $2 \times$ & $4 \times$ & $8 \times$ & $16 \times$ & $32 \times$ & $64 \times$ & $128 \times$ & $256 \times$ & Average \\
& 2 & 4 & 8 & 16 & 32 & 64 & 128 & 256 & \\
\hline Samples & 90 & 90 & 90 & 90 & 90 & 90 & 90 & 90 & 90 \\
$\begin{array}{l}\text { Correct } \\
\text { prediction }\end{array}$ & 83 & 80 & 79 & 78 & 77 & 75 & 78 & 79 & 78.62 \\
Accuracy & 92.2 & 88.8 & 87.7 & 83.3 & 85.5 & 83.3 & 86.6 & 87.7 & 87.36 \\
\hline
\end{tabular}

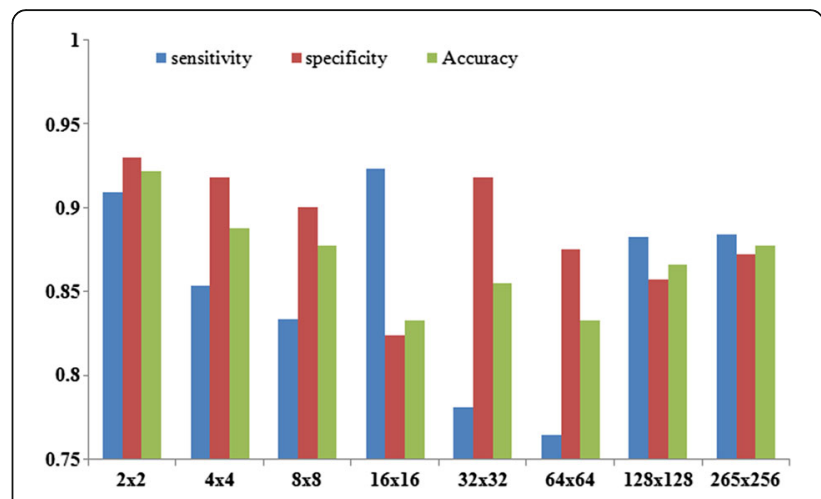

Fig. 7 Relative performance measure for different pixel resolutions

The blue and red colors show the cumulative values for landslide affected images (heavy and medium), whereas green color shows no landslide affected images. The ranges of cumulative values after application of FA for different types of images with $2 \times 2$ pixel resolution are shown in Table 8 . It is observed from the experimental results that the cumulative values for no landslide regions lies between 43 and 85, whereas the cumulative values for heavy landslide region lies between 174 and 203. It is also found that the cumulative values for medium landslide regions lie between 87 and 161 .

\subsubsection{Validation}

In the validation step, 90 landslide images for each pixel resolution $2 \times 2-256 \times 256$ are taken and evaluated in terms of true positive (TP), true negative (TN), false positive (FP), and false negative (FN) values (Tables 9 and 10). From Table 11, it is found that the lowest and the highest accuracies are 92.2 and $83.3 \%$ respectively. For performance evaluation, sensitivity, specificity, accuracy, and the area under the receiver operator curve (ROC) metric are used.

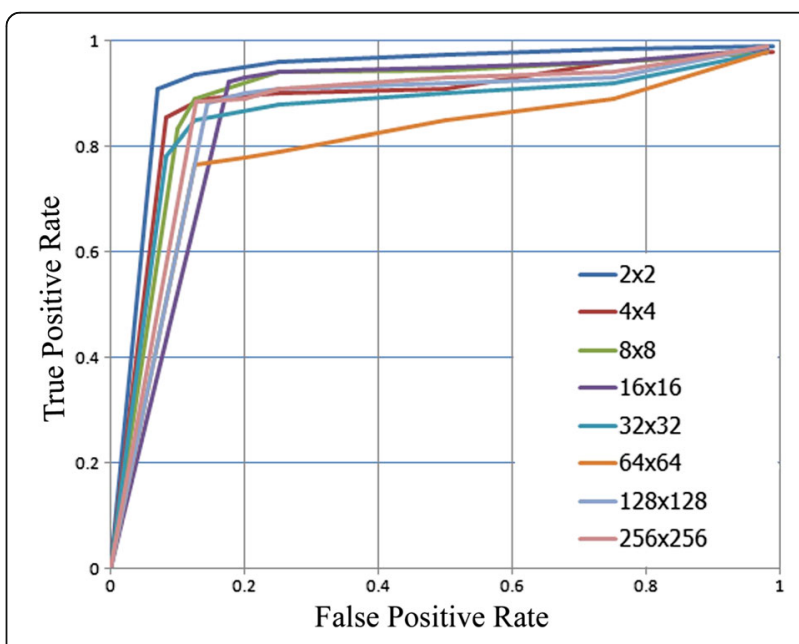

Fig. 8 Variation of area under receiver operator curve (ROC) 
A comparison between accuracy, sensitivity, and specificity is given. The results are plotted in Fig. 7 which depict higher value of accuracy, sensitivity, and specificity. Figure 7 shows better performance of the present study. The performance of the system at different pixel resolutions is evaluated using receiver operating characteristic (ROC) curves $[35,36]$ as shown in Fig. 8. The highest area under the curve (93\%) (Fig. 8) is obtained for $2 \times 2$ pixel resolution. Validation statistics, viz., efficiency, misclassification rate, positive predictive power, negative predictive power, sensitivity, specificity, false positive rate, and false negative rate, are calculated and plotted as shown in Fig. 9. The optimal efficiency $(92.22 \%)$, misclassification rate (7.77\%), negative predictive power $(94.64 \%)$, specificity $(92.98 \%)$, and false positive rate $(7 \%)$ are found in the case of $2 \times 2$ pixel resolution in this study. Optimal value of sensitivity $(92.30 \%)$ and false negative rate $(7.69 \%)$ are considered in the case of $16 \times 16$ pixel resolution.

From the experimental results, it may be concluded that FA performs better compared to the multivariate statistical tools (PCA or LDA) and their hybridized variants. According to Kim [37], PCA or LDA analyzes all of the variance of the set of variables (common variance and unique variance), whereas FA analyzes only common variance (correlation) of the set of variables. The use of PCA and LDA are data reduction by summarizing many variables into a smaller number of components. On the other hand, FA finds a factor model that can reproduce observed correlation; thus, it aimed at explaining the correlation between variables. In hybridized variants of multivariate statistical
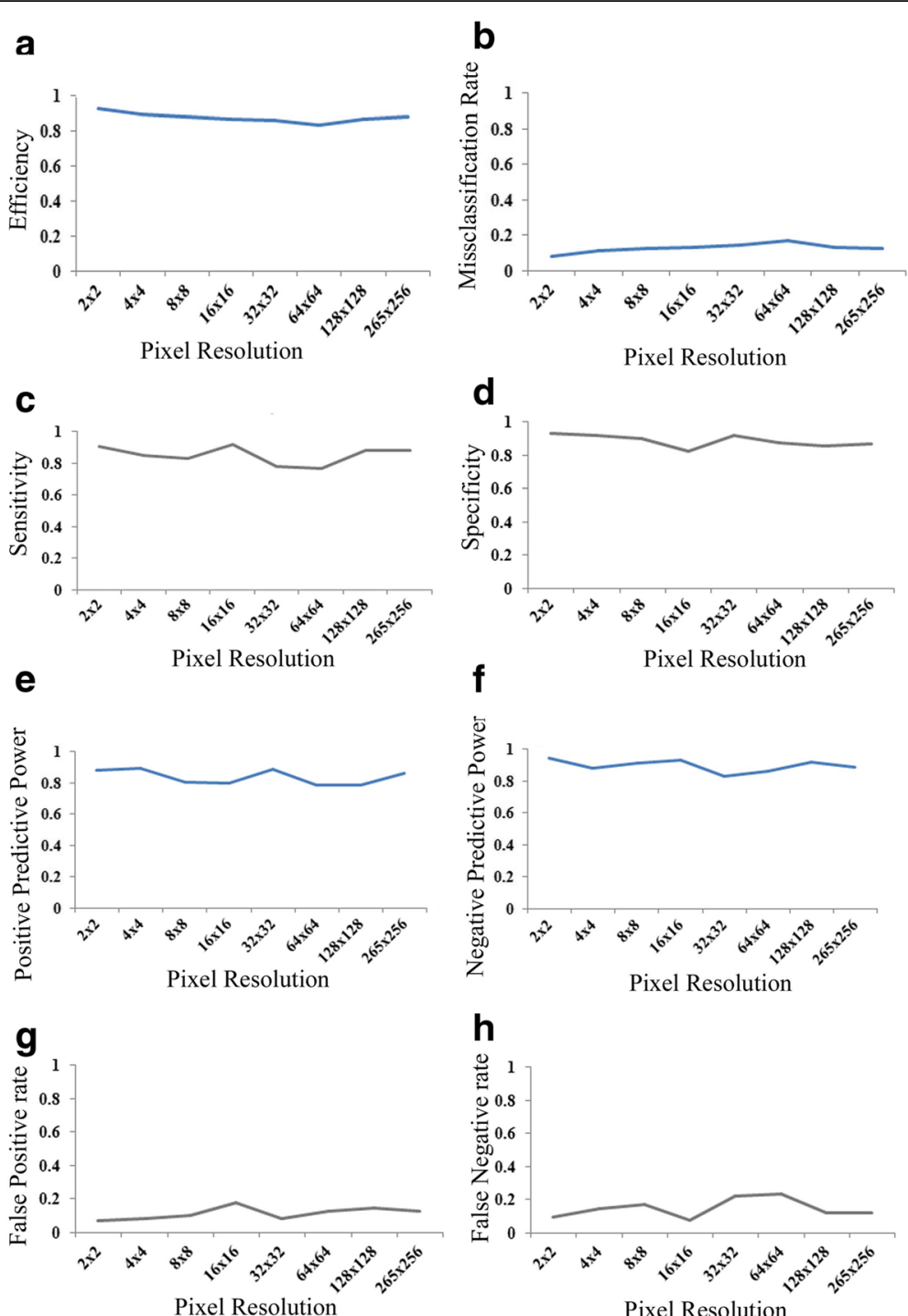

Fig. 9 Validation Statistics. Variation of values of a. efficiency, b. misclassification rate, c. sensitivity, d. specificity, e. positive predictive power, $\mathbf{f}$. negative predictive power, $\mathbf{g}$. false positive rate, $\mathbf{h}$. false negative rate ( $Y$-axis) against different pixel resolutions ( $\mathrm{x}$-axis) 
tools, values arising from PCA or LDA overpower the effect of values arising from FA. So, more deviation of output values is found in hybridized variants of multivariate statistical tools than that of FA.

\section{Conclusions}

In this paper, multivariate statistical tools were used for reducing number of features in order to boost performance of the system with regard to landslide susceptibility analysis. Eight databases were created, and residual analysis was used to decide the best multivariate statistical tools and their hybridized variants. Factor analysis was performed and found to be superior to others and applied thereby. The performance of the system was evaluated using various statistical parameters such as sensitivity, specificity, accuracy, and ROC. The experimental results revealed that the pixel resolution $2 \times 2$ based on factor analysis performs best for heavy and medium landslide images, whereas pixel resolution $8 \times 8$ based on factor analysis came out as optimal for no landslide images. In terms of accuracy also, $2 \times 2$ pixel resolutions show outstanding performance. The developed system can be effectively used for landslide susceptibility analysis for different landslide-affected regions with only satellite images in hand without any physical survey. The validation statistics also prove the robustness of the system can enable analysis of landslide susceptibility of remote locations and holds great prospect for saving lives of human being under the threat of disaster.

\section{Abbreviations \\ AR: Absolute residual; FA: Factor analysis; FD: Fractal dimension; FN: False negative; FP: False positive; GIS: Geographic information system; GLCM: Gray level co-occurrence matrix; LDA: Linear discriminant analysis; MAR: Mean of absolute residual; MMRE: Mean of mean residual error; MRE: Mean residual error; PCA: Principal component analysis; ROC: Receiver operating characteristic; SDAR: Standard deviation of absolute residual; TN: True negative; TP: True positive}

\section{Acknowledgements}

We acknowledge Ikonos Satellite Image, Centre for Remote Imaging, Sensing and Processing, National University of Singapore, for providing satellite images after the landslide of Bukit Antarabangsa via http:// www.crisp.nus.edu.sg.

\section{Funding}

We acknowledge TEQIP-II, Government College of Engineering and Textile Technology, Berhampore, West Bengal, India, for providing financial support.

\section{Authors' contributions}

MIQ designed and tested the proposed algorithm and drafted the manuscript. AH tested the algorithm. MIQ carried out the database creation, application of multivariate statistical tools, and their hybridizations along with in-depth residual analysis. JPC participated in the algorithm design. MIQ performed the performance and statistical analysis. All authors read and approved the final manuscript.

\section{Competing interests}

The authors declare that they have no competing interests.

\section{Author details}

${ }^{1}$ Department of Information Technology, Kalyani Government Engineering College, Kalyani, Nadia, West Bengal, India. ${ }^{2}$ Department of Computer Science \& Engineering, Govt. College of Engineering and Textile Technology, Berhampore, Murshidabad, West Bengal, India.

Received: 3 September 2016 Accepted: 15 February 2017

Published online: 03 March 2017

\section{References}

1. C Tarantino, P Blonda, G Pasquariello, Application of change detection techniques for monitoring man-induced landslide causal factors, IEEE. 2, pp 1103-1106 (2004). 0-7803-8742-2/04.

2. AK Saha, RP Gupta, I Sarkar, MK Arora, E Csaplovics, An approach for GISbased statistical landslide susceptibility zonation - with a case study in the Himalayas. Landslides. 2, 61-69 (2005). doi:10.1007/s10346-004-0039-8

3. L Liang, K Chen, U Chang, J Lien, Monitoring and statistical analysis of landslides in Taiwan Island using multi satellite images and GIS Data, IEEE. 2, pp 1231-1234 (2007). 1-4244-1212-9/07.

4. J Rau, L Chen, J Liu, T Wu, Dynamic monitoring and disaster assessment for watershed management using time-series satellite images, IEEE. 45(6), 01962892 (2007)

5. S Voigt, T Kemper, T Riedlinger, R Kiefl, K Scholte, H Mehl, Satellite image analysis for disaster and crisis-management support, IEEE. 45(6), 0196-2892 (2007).

6. KE Joyce, GD Dellow, PJ Glassey, Assessing image processing techniques for mapping landslides, IGARSS, IEEE. 2, pp 1231-1234 (2008). 978-1-4244-2808-3/08.

7. G Martelloni, S Segoni, R Fanti, F Catani, Rainfall thresholds for the forecasting of landslide occurrence at regional scale. Landslides. 9, 485-495 (2012). doi:10.1007/a10346-011-0308-2

8. DP Kanungo, S Sharma, Rainfall thresholds for prediction of shallow landslides around Chamoli-Joshimath region, Garhwal Himalayas, India. Landslides. 11, 629-638 (2014). doi:10.1007/s10346-013-0438-9

9. K Biro, B Pradhan, M Buchroithner, F Makeschin, Landuse/Land cover change analysis and its impact on soil Properties in the northern part of gadarif region, Sudan, Land Degradation \& Development Wiley online library. (2011). doi: 10.1002/ldr.1116.

10. A Akgun, EA Sezer, HA Nefeslioglu, C Gokceoglu, B Pradhan, An easy-to-use MATLAB program (MamLand) for the assessment of landslide susceptibility using a Mamdani fuzzy algorithm. Computers \& Geosciences. (2011). doi:10.1016/j.cageo.2011.04.012.

11. B Pradhan, S Lee, MF Buchroithner, Use of geospatial data for the development of fuzzy algebraic operators to landslide hazard mapping: a case study in Malaysia. Appl. Geomatic. 1, 3-15 (2009)

12. B Pradhan, AM Youssef, Manifestation of remote sensing data and GIS on landslide hazard analysis using spatial-based statistical models, Arabian. J. Geosciences. (2009). doi:10.1007/s12517-009-0089-2

13. B Pradhan, S Lee, Landslide susceptibility assessment and factor effect analysis: back propagation artificial neural networks and comparison with frequency ratio and bivariate logistic regression modeling. Environ. Model. Softw. 25, 747-759 (2010)

14. S Lee, DG Evangelista, Earthquake induced landslide susceptibility mapping using an artificial neural network. Nat. Hazards. Earth. Syst. Sci. 6, 687-695 (2006)

15. S Lee, Application and verification of fuzzy algebraic operators to landslide susceptibility mapping. Environ. Geol. 52, 615-623 (2007)

16. K Muthu, M Petrou, C Tarantino, P Blonda, Landslide possibility mapping using fuzzy approaches. IEEE. Trans. Geosci. Remote. Sens. 46(4), 1253-1265 (2008). doi:10.1109/tgrs.2007.912441

17. PK Champatiray, S Dimri, RC Lakhera, S Santosh, Fuzzy-based method for landslide hazard assessment in active seismic zone of Himalaya. Landslides. 4, 101-111 (2007)

18. J Li, Q Du, C Sun, An improved box-counting method for image fractal dimension estimation. Pattern. Recogn. 422, 460-2469 (2009). doi:10.1016/j.patcog

19. RM Hawlick, Statistical and structural approaches to texture. Proc. IEEE. 67(5), 786-804 (1979)

20. R Thangavel, R Manavalan, Soft computing models based feature selection for TRUS prostate cancer image classification. Soft. Comput. 18, 1165-1176 (2014). doi:10.1007/s00500-013-1135-2

21. V Vakharia, VK Gupta, PK Kankar, A comparison of feature ranking techniques for fault diagnosis of ball bearing. Soft. Comput. 20, 1601-1619 (2016). doi:10.1007/s00500-015-1608-6 
22. C Fernandez-Lozano, JA Seoane, M Gestal, TR Gaunt, J Dorado, C Campbell, Texture classification using feature selection and kernel based technique. Soft. Comput. 19, 2469-2480 (2015). doi:10.1007/s00500-014-1573-5

23. M Mercimek, K Gulez, TV Mumcu, Real object recognition using moment invariants. Sadhan. 30(6), 765-775 (2005)

24. MNB Filho, FJA Sobreira, Accuracy of lacunarity algorithms in texture classification of high spatial resolution images from urban areas, The International Archives of the Photogrammetry. Remote Sensing and Spatial Information Sciences. vxxxvii(b3b), pp 417-422 (2008).

25. B Fernando, E Fromont, T Tuytelaars, Mining mid-level features for image classification. Int. J. Comput. Vis. 108, 186-203 (2014). doi:10.1007/s11263014-0700-1

26. DG Lowe, Distinctive image features from scale-invariant keypoints. Int. J. Comput. Vis. 60(2), 91-110 (2004)

27. AC Rencher, Methods of Multivariate Analysis, 2nd Edn. (John Willey, USA, 2002), pp 102-105.

28. R Doerffer, D Murphy, Factor analysis and classification of remotely sensed data for monitoring tidal flats. Helgolander. Meeresunters. 43, 275-293 (1989)

29. C Rodarmel, J Shan, Principal component analysis for hyperspectral image classification. Surveying. Land. Inf. Syst. 62(2), 115-123 (2002)

30. RA Johnson, DW Wichern, Applied Multivariate analysis, 6th Edn. (Pearson Prentice Hall, New Jersey, 2007), pp 102-110.

31. C Yan, Y Zhang, J Xu, F Dai, J Zhang, Q Dai, F Wu, Efficient parallel framework for HEVC motion estimation on many-core processors. IEEE. Trans. Circuits. Syst. Video. Technol. 24(12), 2077-2089 (2014)

32. C Yan, Y Zhang, J Xu, F Dai, L Li, Q Dai, F Wu, A highly parallel framework for HEVC coding unit partitioning tree decision on many-core processors. IEEE. Signal. Process. Lett. 21(5), 573-576 (2014)

33. Ikonos Satellite Image, Centre for Remote Imaging, Sensing and Processing, National University of Singapore. http://www.crisp.nus.edu.sg. Accessed 20 Mar 2016.

34. TH Low, Area based landslide hazard assessment for hillside development, (Ph. D thesis, University of Malaya, 2011)

35. E Yesilnacar, T Topal, Landslide susceptibility mapping: a comparison of logistic regression and neural networks methods in medium scale study, Hendek region, Turkey. Eng. Geol. 79, 251-266 (2005). doi:10.1016/j.enggeo. 2005.02.002

36. JA Swets, Measuring the accuracy of the diagnostic systems. Science. 240, 1285-1293 (1988). doi:10.1126/science.3287615.

37. HJ Kim, Common factor analysis vs principal component analysis: choice for symptom cluster research. Asian. Nurs. Res. 2(1), 17-24 (2008)

\section{Submit your manuscript to a SpringerOpen ${ }^{\circ}$ journal and benefit from:}

- Convenient online submission

$\checkmark$ Rigorous peer review

- Immediate publication on acceptance

- Open access: articles freely available online

- High visibility within the field

- Retaining the copyright to your article 